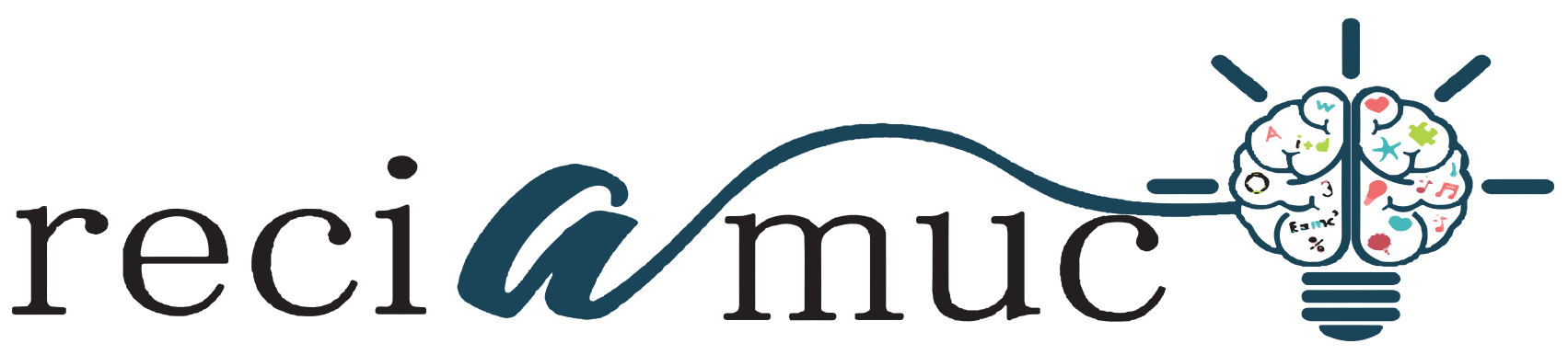

DOI: 10.26820/reciamuc/4.(4).noviembre.2020.198-206

URL: https://reciamuc.com/index.php/RECIAMUC/article/view/554

EDITORIAL: Saberes del Conocimiento

REVISTA: RECIAMUC

ISSN: 2588-0748

TIPO DE INVESTIGACIÓN: Artículo de Revisión

CÓDIGO UNESCO: 32 Ciencias Médicas

PAGINAS: $198-206$

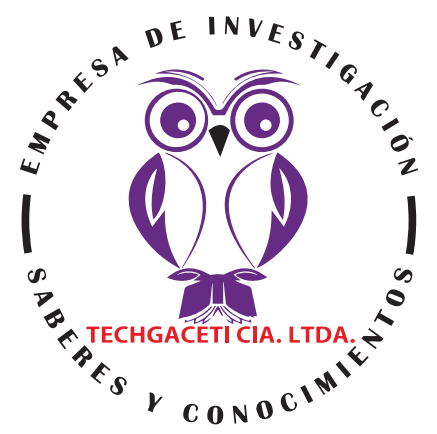

\title{
El estrés psicosocial como causa de gastritis
}

Psychosocial stress as a cause of gastritis

0 stress psicossocial como causa de gastrite

\section{Jessenia Paola Ochoa Bustamante'; Leonel Amador Zúñiga Arreaga2; Janeth Mabel Rojas Riera3; Daniel David Figueroa Contreras ${ }^{4}$}

RECIBIDO: 18/07/2020 ACEPTADO: 20/09/2020 PUBLICADO: 30/11/2020

1. Master Universitario en Prevención de Riesgos Laborales; Obstetra; Médico; Investigadora Independiente; Guayaquil, Ecuador; pao_ochoab@hotmail.com; (iD) https://orcid.org/0000-0003-2069-0371

2. Magister en Gerencia de Seguridad y Salud en el Trabajo; Magister en Epidemiologia; Especialista en Salud y Seguridad Ocupacional Mención en Salud Ocupacional; Licenciado en Laboratorio Clínico; Médico; Investigador Independiente; Guayaquil, Ecuador; dr.leonelzuniga@outlook.com; (iD https://orcid.org/0000-0003-2666-3905

3. Especialista en Medicina Familiar y Comunitaria; Médico; Investigadora Independiente; Guayaquil, Ecuador; mabelina87@ gmail.com; (D) https://orcid.org/0000-0002-1612-760X

4. Magister en Gerencia y Administración de Salud; Médico; Investigador Independiente;Guayaquil, Ecuador; danieldfigueroa@hotmail.com; iD https://orcid.org/0000-0002-0250-6246

CORRESPONDENCIA

Jessenia Paola Ochoa Bustamante

pao_ochoab@hotmail.com

Guayaquil, Ecuador

(C) RECIAMUC; Editorial Saberes del Conocimiento, 2020 


\section{RESUMEN}

La gastritis es un tipo de enfermedad que en ocasiones está asociada con el estrés considerándose como una lesión grave con presencia de sepsis e insuficiencia orgánica. Este tipo de afección, también es llamada gastritis emotiva o gastritis nerviosa producto del estrés y la ansiedad que pueda existir en el paciente que la padece, los síntomas son muy similares a los de la gastritis bacteriana. Presenta mayor incidencia en la población adulta, sin embargo, su incidencia ha disminuido durante la última década Los factores que contribuyen a esta disminución incluyen el transporte más rápido de los pacientes traumatizados, la reanimación temprana, el tratamiento de complicaciones y el mantenimiento profiláctico del aumento del pH de la mucosa gástrica. La fisiopatología queda por aclararse por completo; no obstante, tanto factores agresivos (ácido, reflujo duodenal, etc.) como una deficiencia en los mecanismos defensivos (flujo sanguíneo de la mucosa gástrica, barrera de la mucosa gástrica, moco, bicarbonato, etc.) juegan un papel importante en su inicio. La complicación hemorrágica de la úlcera gástrica por estrés, que suele aparecer entre el quinto y el décimo día tras el ingreso, sigue siendo una secuela asociada a una importante tasa de mortalidad.

Palabras clave: Gastritis, Estrés, Ulcera gástrica.

\section{ABSTRACT}

Gastritis is a type of disease that is sometimes associated with stress and is considered a serious injury with the presence of sepsis and organ failure. This type of condition is also called emotional gastritis or nervous gastritis product of the stress and anxiety that may exist in the patient who suffers from it, the symptoms are very similar to those of bacterial gastritis. It has a higher incidence in the adult population, however, its incidence has decreased over the last decade. Factors that contribute to this decrease include faster transport of trauma patients, early resuscitation, treatment of complications, and prophylactic maintenance of the increase of the $\mathrm{pH}$ of the gastric mucosa. The pathophysiology remains to be fully clarified; However, both aggressive factors (acid, duodenal reflux, etc.) and a deficiency in the defensive mechanisms (blood flow of the gastric mucosa, gastric mucosa barrier, mucus, bicarbonate, etc.) play an important role in its onset. . The hemorrhagic complication of gastric stress ulcer, which usually appears between the fifth and tenth day after admission, continues to be a sequela associated with a significant mortality rate.

Keywords: Gastritis, Stress, Gastric ulcer.

\section{RESUMO}

A gastrite é um tipo de doença que está por vezes associada ao stress e é considerada uma lesão grave com a presença de septicemia e falência de órgãos. Este tipo de doença é também chamado gastrite emocional ou gastrite nervosa produto do stress e da ansiedade que pode existir no paciente que sofre dela, os sintomas são muito semelhantes aos da gastrite bacteriana. Tem uma maior incidência na população adulta, contudo, a sua incidência diminuiu ao longo da última década. Os factores que contribuem para esta diminuição incluem o transporte mais rápido dos pacientes traumatizados, a reanimação precoce, o tratamento de complicações e a manutenção profiláctica do aumento do pH da mucosa gástrica. A fisiopatologia continua por esclarecer; contudo, tanto os factores agressivos (ácido, refluxo duodenal, etc.) como uma deficiência nos mecanismos defensivos (fluxo sanguíneo da mucosa gástrica, barreira da mucosa gástrica, muco, bicarbonato, etc.) desempenham um papel importante no seu início. . A complicação hemorrágica da úlcera de stress gástrico, que geralmente aparece entre o quinto e o décimo dia após a admissão, continua a ser uma sequela associada a uma taxa de mortalidade significativa.

Palavras-chave: Gastrite, Stress, Úlcera gástrica. 


\section{Introducción}

La producción de ácido gástrico es necesaria para que el cuerpo digiera los alimentos y descomponga los componentes nutricionales en aminoácidos, carbohidratos y grasas absorbibles. La mayor parte del ácido se produce cuando el pH gástrico estimula la liberación y activación de varias enzimas digestivas. El estómago es un ambiente relativamente ácido con un $\mathrm{pH}$ de menos de 4.0 , y este puede caer a 2.0 con la presencia de células parietales. "Las células parietales viven en el fondo de ojo y el cuerpo del estómago y secretan iones de hidrógeno. La secreción de iones de hidrógeno es estimulada por tres sustancias predominantes" (Saavedra \& Benicky, 2007).

1. Neurotransmisor acetilcolina $(\mathrm{ACH})$ estimula las células parietales a través de la vía de la fosfolipasa para secretar hidrógeno. La fosfolipasa se estimula en la pared celular, que luego escinde PIP en IP3 y DAG, liberando iones de calcio en el citoplasma. Estos iones de calcio se unen a calmodulina y estimulan la proteína quinasa $\mathrm{C}$ (PKC). La PKC conduce a la fosforilación y activación de la ATPasa de hidrógeno / potasio (H / K ATPasa), lo que resulta en la secreción de ácido. La fuente de $\mathrm{ACH}$ es el nervio vago (par craneal $X)$.

2. La gastrina es una hormona conocida por estimular la secreción de iones de hidrógeno a través del mismo mecanismo de la $\mathrm{ACH}$, lo que resulta en la activación de la ATPasa H / K. La gastrina se secreta predominantemente a través de las células $G$, ubicadas en el antro del estómago, que son estimuladas por la presencia de aminoácidos y $\mathrm{ACH}$ en la luz gástrica.

3. La histamina estimula a las células parietales a secretar iones de hidrógeno a través de la vía cAMP a través de la activación de la proteína quinasa $A$ y activar la ATPasa H / K. La histamina se secreta predominantemente a través de los mastocitos de los tejidos gástricos circundantes.

La desregulación de los mecanismos mencionados anteriormente puede resultar en gastropatía hemorrágica o erosiva, también conocida como gastritis por estrés. La barrera mucosa se rompe como consecuencia de una enfermedad aguda. El pH alterado puede interrumpir la digestión homeostática, lo que resulta en una desregulación del pH gástrico.

En el entorno clínico, esta desregulación se debe típicamente al estrés fisiológico, que puede provocar una inflamación del estómago, conocida como gastritis inducida por estrés. En el estado de estrés, los niveles elevados de $\mathrm{ACH}$ e histamina dan como resultado un aumento de la producción de ácido y, por lo tanto, inducen gastritis (Auguste \& Lackner, 2010)

Los pacientes se encuentran típicamente en la unidad quirúrgica del hospital o en la unidad de cuidados intensivos médicos o quirúrgicos. Estos pacientes críticamente enfermos han sufrido típico estrés fisiológico relacionado con traumatismos graves, quemaduras graves, dependencia del ventilador o traumatismos intracraneales. De acuerdo con Rodríguez, (2017) "el estrés fisiológico que conduce a la gastritis por estrés produce erosiones gástricas, conocidas como úlceras que se encrespan. Las erosiones secundarias a la etiología craneal se denominan úlceras de Cushing en honor al famoso neurocirujano Harvey Cushing" (p. 22)

El primer paso en el desarrollo de la gastritis inducida por estrés es la disminución de la resistencia de la mucosa a los radicales tóxicos. "La respuesta al estrés del cuerpo da como resultado la disminución de la renovación gástrica, lo que conduce a la atrofia de la mucosa gástrica" (Rodríguez, 2017). El flujo de sangre al estómago disminuye y hace que el estómago sea más propenso a la ulceración de ácido-pepsina, así como a la secreción de hiperácido. 
Esta investigación tiene como objetivo discutir la etiología y los métodos para prevenir e identificar la gastritis por estrés con la finalidad de brindar información de interés para futuras investigaciones.

\section{Metodología}

Para el desarrollo de este proceso investigativo, se plantea como metodología la encaminada hacia una orientación científica particular que se encuentra determinada por la necesidad de indagar en forma precisa y coherente una situación, en tal sentido Davila, (2015) define la metodología "como aquellos pasos anteriores que son seleccionados por el investigador para lograr resultados favorables que le ayuden a plantear nuevas ideas" (p.66)

Lo citado por el autor, lleva a entender que el desarrollo de la acción investigativa busca simplemente coordinar acciones enmarcadas en una revisión bibliográfica con el fin de complementar ideas previas relacionadas El estrés psicosocial como causa de gastritis a través de una revisión de literatura, para así finalmente elaborar un cuerpo de consideraciones generales que ayuden a ampliar el interés propuesto.

\section{Tipo de Investigación}

Dentro de toda práctica investigativa, se precisan acciones de carácter metodológico mediante las cuales se logra conocer y proyectar los eventos posibles que la determinan. En este sentido, la presente investigación corresponde al tipo documental, definido por Castro (2016), "se ocupa del estudio de problemas planteados a nivel teórico, la información requerida para abordarlos se encuentra básicamente en materiales impresos, audiovisuales y / o electrónicos". (p.41).

En consideración a esta definición, la orientación metodológica incluye la oportunidad de cumplir con una serie de actividades inherentes a la revisión y lectura de diversos documentos, donde se encuentran ideas explicitas relacionadas con los tópicos encargados de identificar una característica inmersa en el estudio. Por lo tanto, se realizaron continuas interpretaciones con el claro propósito de revisar aquellas apreciaciones propuestas por diferentes investigadores en relación al tema de interés, para luego dar la respectiva argumentación a los planteamientos, en función a las necesidades encontradas en la investigación, apoyados en las herramientas tecnológicas para la búsqueda de trabajos con valor científico disponibles en la web que tenían conexión con el objetivo principal de la investigación.

\section{Fuentes Documentales}

El análisis correspondiente a las características que predomina en el tema seleccionado, llevan a incluir diferentes fuentes documentales encargadas de darle el respectivo valor científico y en ese sentido cumplir con la valoración de los hechos a fin de generar nuevos criterios que sirven de referencia a otros procesos investigativos. Para Castro,(2016) las fuentes documentales incorporadas en la investigación documental o bibliográfica, "representa la suma de materiales sistemáticos que son revisados en forma rigurosa y profunda para llegar a un análisis del fenómeno" (p.41). Por lo tanto, se procedió a cumplir con la lectura previa determinada para encontrar aquellos aspectos estrechamente vinculados con el tema, con el fin de explicar mediante un desarrollo las respectivas apreciaciones generales de importancia.

\section{Técnicas para la Recolección de la Infor- mación}

La conducción de la investigación para ser realizada en función a las particularidades que determinan a los estudios documentales, tiene como fin el desarrollo de un conjunto de acciones encargadas de llevar a la selección de técnicas estrechamente vinculadas con las características del estudio. Bolívar, (2015), refiere, que es "una técnica particular para aportar ayuda a los procedimientos de selección de las ideas primarias

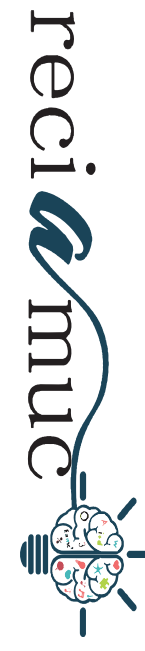


y secundarias". (p.71).

Tal como lo expresa, Bolívar, (2015) "Las técnicas documentales proporcionan las herramientas esenciales y determinantes para responder a los objetivos formulados y llegar a resultados efectivos" (p. 58). Es decir, para responder con eficiencia a las necesidades investigativas, se introdujeron como técnica de recolección el método inductivo, que hizo posible llevar a cabo una valoración de los hechos de forma particular para llegar a la explicación desde una visión general. El autor Bolívar, (2015) tambien expresa que las técnicas de procesamiento de datos en los estudios documentales "son las encargadas de ofrecer al investigador la visión o pasos que deben cumplir durante su ejercicio, cada una de ellas debe estar en correspondencia con el nivel a emplear" (p. 123). Esto indica, que para llevar a cabo el procesamiento de los datos obtenidos una vez aplicadas las técnicas seleccionadas, tales como: fichas de resumen, textual, registros descriptivos entre otros, los mismos se deben ajustar al nivel que ha sido seleccionado.

\section{Resultados}

\section{Epidemiología}

Los autores Wuerth, Thomas, \& Tolia, (2008) "informaron que la incidencia de hemorragia gastrointestinal superior es de aproximadamente 81 casos por 100.000; este número se redujo a 67 casos por 100.000 en 2012". Se encontró que la mayor disminución se registró en la población con gastritis y úlcera péptica.

Su disminución fue del 55\% y 30\% respectivamente, y probablemente se atribuyó a la institución y al uso temprano de inhibidores de la bomba de protones (IBP) o bloqueadores de histamina. Las estimaciones de la forma asintomática de gastritis, en pacientes críticamente enfermos, que no están recibiendo profilaxis son altas (aproximadamente> 75 por ciento). (Wuerth, Thomas, \& Tolia, 2008)
La úlcera gástrica por estrés con hemorragia oculta tiene una tasa de incidencia del 15 al 50 por ciento, la hemorragia manifiesta es del 1,5 al 8,5 por ciento y la úlcera gástrica por estrés con hemorragia clínicamente significativa es incluso baja, del 1 al 3 por ciento.

\section{Fisiopatología}

El aumento de la secreción de ácido conduce al desarrollo de erosiones que pueden provocar hemorragia gástrica. Aunque estas hemorragias pueden no poner en peligro la vida inicialmente, pueden causar malestar al paciente o deposiciones melanóticas en la fase inicial y hemorragia grave 4 a 5 días después.

El estrés provoca la liberación de angiotensina II, que disminuye el flujo sanguíneo a la mucosa.

Esto provoca la formación de especies reactivas de oxígeno, que atacan al ADN y dan como resultado la formación de 8-hidroxidesoxiguanosina (8-OHdG). Lo que da como resultado un subproducto mutagénico oxidativo y, posteriormente, estrés oxidativo en la mucosa. Por otro lado, se cree que el óxido nítrico producido naturalmente protege contra la gastritis por estrés porque promueve la vasodilatación. (Rodríguez, 2017)

Los síntomas iniciales pueden ser náuseas persistentes asociadas con dolor epigástrico, pero la hemorragia suele ser el primer síntoma. Para un paciente en la unidad de cuidados intensivos, la salida de la sonda nasogástrica puede volverse sanguinolenta. Un paciente puede incluso desarrollar hematemesis.

La esofagogastroduodenoscopia (EGD) puede mostrar inflamación gástrica difusa y erosiones leves en el estómago y el duodeno. "Estas úlceras tienden a ser superficiales. Por lo general, las erosiones gástricas agudas en pacientes con quemaduras o traumatismos graves se pueden observar en los 3 días posteriores a la lesión" (Tryba 
\& May, 2002). El síntoma de presentación más común de la gastritis inducida por estrés es una hemorragia. Raramente, aproximadamente el 10\%, los pacientes desarrollarán perforación.

\section{Histopatología}

La gastritis se caracteriza "por inflamación, edema mucoso, dilatación vascular, proliferación de músculo liso en la lámina propia y congestión vascular. Las glándulas de la mucosa gástrica pueden parecer tortuosas o adquirir una apariencia de sacacorchos" (Wuerth, Thomas, \& Tolia, 2008). De acuerdo con MacDonald, (2006) "hay infiltración neutrofílica y granulación activa con infiltración mononuclear dentro de las capas mucosas. También se puede encontrar necrosis fibrinoide". En las formas crónicas, se observa que los linfocitos, los monocitos y las células plasmáticas se infiltran en la mucosa y submucosa.

\section{Causas de la gastritis por estrés}

Considerando que las enfermedades psicosomáticas tienen una relación directa con determinadas actitudes emocionales, se debe mencionar, que la gastritis representa una de este tipo de enfermedades, probablemente por el estrés que puedan generar las responsabilidades familiares, sociales o laborales en el individuo. Esta afección, puede presentarse temporalmente en el paciente durante un corto periodo de tiempo o prolongarse debido a las características de personalidad de quien la padece, Ilegando a desencadenar problemas graves e incluso la muerte. En tal sentido, Martínez, (2020) puntualiza las principales causas de la gastritis por estrés, entre las que se mencionan:

- Estrés

- Ansiedad

- Nerviosismo

- Tensión emocional

- Agobio
- Frustración

\section{Anamnesis y examen físico}

Un paciente que se presenta con gastritis inducida por estrés por lo general ha sufrido un evento fisiológico estresante reciente, como politraumatismo severo por una colisión significativa de vehículo motorizado o una caída que resulta en múltiples fracturas de huesos.

La gastritis inducida por estrés también puede ser provocada por una enfermedad grave, como una neumonía dependiente del ventilador o un infarto de miocardio masivo. En algunos casos los pacientes pueden presentar gastritis por estrés después de una cirugía mayor. Es pertinente mencionar que algunas formas de estresores psiquiátricos, como la depresión mayor no tratada, pueden resultar en gastritis por estrés. (Rodríguez, 2017)

El examen físico puede mostrar signos vitales estables o inestables, según la agresión provocada. Si un paciente se ha sometido a una cirugía mayor, los signos vitales pueden estar estables; sin embargo, el estrés al cuerpo aún puede ser grande. Auguste \& Lackner, (2010) exponen "en un paciente que experimentó un infarto de miocardio masivo, cabría esperar signos vitales menos estables. Por último, se esperaría que un paciente con politraumatismo e inestabilidad hemodinámica presente signos vitales muy inestables hasta su reanimación". Los siguientes signos y síntomas están presentes en los casos típicos:

- Vómito molido de café

- Hematemesis

- Melena

- Dolor abdominal

- Náuseas

- Ortostasis en casos severos

- Evaluación 
Si se coloca una sonda nasogástrica, después de enjuagar con solución salina normal, el retorno puede estar teñido de sangre. Generalmente, "se puede descartar una hemorragia digestiva alta después de la colocación de una sonda nasogástrica y el lavado gástrico devuelve contenido bilioso, también se realiza una prueba de sangre oculta en heces (FOBT)" (Saavedra \& Benicky, 2007). La endoscopia se usa para hacer un diagnóstico definitivo.

La gastritis por estrés se observa como pequeñas erosiones superficiales de la mucosa o lesiones eritematosas en el cuerpo y el fondo gástrico. Aunado a ello, se pueden realizar pruebas para la infección por $\mathrm{H}$. pylori, como la prueba del aliento con ureasa o la prueba del antígeno en heces. (Rodríguez, 2017)

\section{Tratamiento y manejo}

El inicio de cuidados intensivos mejorados ha disminuido la incidencia de gastritis inducida por estrés. "El pilar del tratamiento es la administración de IBP los cuales son medicamentos que funcionan al reducir la cantidad de ácido producido por glándulas en el revestimiento del estómago y la segunda línea es el uso de bloqueadores de histamina" (Knodell, Garjian, \& Schreiber, 2007).

Los IBP, los fármacos "azólicos" como el omeprazol y el lansoprazol, son inhibidores irreversibles de la ATPasa H / K. "Esto da como resultado una disminución de la secreción de iones de hidrógeno independientemente de los niveles de acetilcolina y gastrina, ya que el fármaco inhibe directamente la enzima" (Peura \& Johnson, 2005). Los pacientes que reciben IBP desarrollarán una elevación paradójica de la gastrina. Debido a esto, si se suspende los inhibidores de la bomba de protones (PPI), este paciente desarrollará un aumento agudo en la producción de ácido. Esto es secundario a la pérdida de inhibición de la ATPasa $H$ / $\mathrm{K}$, con un nivel de gastrina artificialmente elevado.
Los bloqueadores $\mathrm{H} 2$, como la famotidina, no afectan directamente a la ATPasa H / K de las células parietales. Estos funcionan para inhibir la estimulación de las células parietales mediada por histamina, lo que resulta en una disminución de la fosforilación y activación de la H / K ATPasa a través de la proteína quinasa $A$. (Peura \& Johnson, 2005)

Para esto, es imperativa la administración temprana de inhibidores de la secreción de ácido en forma de PPI y $\mathrm{H} 2$ que son bloqueadores en pacientes críticamente enfermos para prevenir la gastritis inducida por estrés. Dado que la etiología del estrés gástrico proviene de la angiotensina, se están realizando estudios sobre cómo reducir los efectos de la angiotensina en la mucosa gástrica.

\section{Diagnóstico diferencial}

La autora Rodríguez, (2017) expone que lo siguiente también debe ser considerado en el diagnóstico diferencial:

- Enfermedad ulcerosa péptica

- Antinflamatorio no esteroideo (AINE) inducida por gastritis

- Gastropatía alcohólica

- Enfermedad por reflujo gastroesofágico (GERD)

- Cáncer de esófago

- Gastroparesis

- Cáncer pancreático

- Dolor Biliar

- Gastropatía urémica

- Dispepsia

\section{Estudios pertinentes y ensayos en curso}

Los inhibidores de la bomba de protones (IBP) siempre se han considerado superiores a los bloqueadores del receptor $2(\mathrm{H} 2)$ de histamina para prevenir la gastritis rela- 
cionada con el estrés en pacientes críticamente enfermos en riesgo.

Estudios recientes han planteado la preocupación por el aumento de la mortalidad en pacientes que reciben IBP para la profilaxis de úlceras por estrés. Faltan ensayos grandes que comparen directamente los agentes para la profilaxis de las úlceras por estrés y se necesitan más estudios para determinar si los IBP son perjudiciales. (Rodríguez, 2017)

Los datos también sugieren el papel potencial de la angiotensina en la gastritis, y se están realizando estudios sobre cómo reducir los efectos de la angiotensina en la mucosa gástrica.

\section{Pronóstico}

El pronóstico depende predominantemente de la gravedad del evento desencadenante. Sin embargo, si el paciente está hemodinámicamente estable y no se tiene en cuenta la profilaxis con PPI o bloqueador de histamina, puede ocurrir una hemorragia intestinal potencialmente mortal, seguida de perforación, shock séptico resultante y posible muerte. "Si se emplean medidas profilácticas agresivas para la población adecuada de pacientes con riesgo de desarrollar úlceras por estrés, el pronóstico suele ser favorable en la mayoría de las circunstancias" (Peura \& Johnson, 2005).

\section{Complicaciones}

La ulceración por estrés puede estar asociada con las siguientes complicaciones: Sangrado, anemia, estenosis, perforación, peritonitis, fístula gastro-cólica debido a perforación de la úlcera, obstrucción de la salida gástrica debido a estenosis, choque hemorrágico, aumento de la estancia en la $\mathrm{UCl}$ y la muerte.

\section{Consultas}

Los pacientes con gastritis relacionada con el estrés generalmente se tratan en unidades de cuidados intensivos. Se debe con- sultar al servicio de gastroenterología si existen signos o síntomas relacionados con el desarrollo de úlceras. También se debe consultar con los equipos de neurocirugía y cirugía general si surge gastritis por estrés en el contexto de una lesión en la cabeza o bollos, respectivamente.

El trabajo en equipo interprofesional mejora los resultados de mortalidad y morbilidad en el contexto de la ulceración por estrés. Los servicios de farmacia juegan un papel clave en la determinación de las dosis adecuadas de medicamentos, especialmente en pacientes con otras comorbilidades importantes (Laudanno, 2007).

\section{Disuasión y educación de los pacientes}

Como se ha visto, la gastritis por estrés se define como llagas en el tracto digestivo que pueden causar malestar estomacal y provocar sangrado; con síntomas que incluyen dolor abdominal superior, náuseas, vómitos o sangre en las heces.

En situaciones estresantes, hay un exceso de ácido en el sistema y la capa protectora de moco en el revestimiento se rompe, lo que lo hace más susceptible al daño. El tratamiento profiláctico en las unidades de cuidados intensivos conduce a la disminución de la recurrencia de las úlceras por estrés. (MacDonald, 2006).

Los proveedores de atención médica son la mejor fuente de información para las inquietudes relacionadas con la formación de úlceras relacionadas con el estrés.

\section{Conclusiones}

Durante el desarrollo del proceso investigativo se observó, que existen evidencias de que el estrés psicosocial tenga relación con la aparición de gastritis en algunos individuos, sin embargo, no se considera un factor único para la aparición de este tipo de afectación. No obstante, la gastritis inducida por estrés se diagnostica comúnmente en clínicas y es tratada como parte de la atención primaria, por el médico o especia-

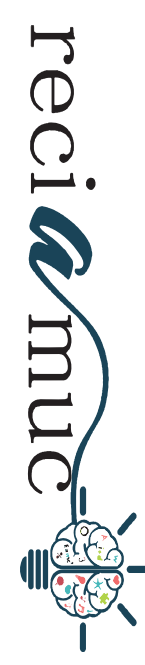


lista del departamento de emergencias o de consulta externa.

Para su diagnóstico se evalúa la historia del paciente y se trata empíricamente con inhibidores de la bomba de protones (IBP). Si los síntomas desaparecen, se insta al paciente a que adopte medidas para aliviar el estrés, entre las que se mencionan: dejar de fumar, disminuir el consumo de las bebidas con cafeína, del alcohol y antinflamatorios no esteroideos (AINEs).

Desafortunadamente, la mayoría de los pacientes siguen sin cumplir con los métodos preventivos y tienen una recurrencia de los síntomas con regularidad. Algunos de estos pacientes pueden beneficiarse de la endoscopia digestiva alta para descartar un trastorno orgánico. La terapia con IBP a largo plazo durante más de 12 meses no está recomendada, en su lugar se prefiere el uso de bloqueadores del receptor $2(\mathrm{H} 2)$ de histamina.

\section{Bibliografía}

Auguste, L., \& Lackner, R. (2010). Prevention of stress-induced erosive gastritis by parenteral administration of arachidonic acid. JPEN J Parenter Enteral Nutr, 615-7.

Knodell, R., Garjian, P., \& Schreiber, J. (2007). Newer agents available for treatment of stress-related upper gastrointestinal tract mucosal damage. Am J Med, 36-40.

Laudanno, O. (2007). Cytoprotective effect of S-adenosylmethionine compared with that of misoprostol against ethanol-, aspirin-, and stress-induced gastric damage. Am J Med, 43-7.

MacDonald, A. (2006). Treatment of stress-induced upper gastrointestinal/hemorrhage with metiamide. Lancet, 68-70.

Martínez, E. (24 de Julio de 2020). Gastritis nerviosa. Mejor con salud.

Peura, D., \& Johnson, L. (2005). Cimetidine for prevention and treatment of gastroduodenal mucosal lesions in patients in an intensive care unit. Ann Intern Med, 173-7.

Rodríguez, A. (2017). Causas psicosociales de la úlcera gastroduodenal. Revista Digital de Medicina Psicosomática y Psicoterapia, 11(2), 1-22.

Saavedra, J., \& Benicky, J. (2007). Brain and peripheral angiotensin II play a major role in stress. Stress, 185-93.

Tryba, M., \& May, B. (2002). Conservative treatment of stress ulcer bleeding: a new approach. Scand J Gastroenterol Suppl, 16-24.

Wuerth, A., Thomas, R., \& Tolia, V. (2008). Gastroesophageal reflux disease and dental erosion in children. J Pediatr, 474-8.

\section{CITAR ESTE ARTICULO:}

Ochoa Bustamante, J. P., Zúñiga Arreaga, L. A., Rojas Riera, J. M., \& Figueroa Contreras, D. D. (2020). Estrés psicosocial como causa de gastritis. RECIAMUC, 4(4), 198-206. https://doi.org/10.26820/reciamuc/4.(4).noviembre.2020.198-206 